\title{
Water and Chlorophyll Content and Leaf Anatomy of Patchouli Planlet (Pogostemon cablin Benth.) Resulted by Shoot-tip Culture Experience Hyperhydricity after Treatment of Modification Ammonium nitrate or Macro salt Concentration on MS medium (Murashige Skoog)
}

\author{
Aisyah Rahmawati Zen ${ }^{1}$, Wahyu Widoretno ${ }^{2}$, Serafinah Indriyani ${ }^{3}$ \\ ${ }^{1}$ Master Program of Biology, Faculty of Mathematics and Natural Sciences, University of Brawijaya, Malang, Indonesia \\ ${ }^{2}$ Laboratory of Plant Culture Physiology and Microtechnique, University of Brawijaya, Malang, Indonesia \\ ${ }^{3}$ Laboratory of Plant Taxonomy, University of Brawijaya, Malang, Indonesia
}

\begin{abstract}
Hyperhydricity is a symptom of abnormal morphological and physiological function which inhibits the regeneration of plantlets. In general, the main symptom of hyperhydricity is a change in the condition of the plantlets which looks clear (Glassy) as a result of low levels of chlorophyll, the high water content in the plantlets, and the abnormal anatomical structure of the leaves. Hyperhydricity can be controlled by reducing cytokinin concentration, increasing gelling agent concentration, and reducing ammonium nitrate and macro salt concentration on medium. Objective of this research was to reduce hyperhydricity in shoot tip culture of patchouli by modification of ammonium nitrate and macro salt concentrtion on MS medium. The various treatment concentrations of ammonium nitrate were $0 \mathrm{mg} \cdot \mathrm{L}^{-1}(0), 41.25 \mathrm{mg} \cdot \mathrm{L}^{-1}(1 / 4$ concentration), $825 \mathrm{mg} \cdot \mathrm{L}^{-1}$ ( $1 / 2$ concentration), $1650 \mathrm{mg}^{-\mathrm{L}^{-1}}$ (1 concentration) and macro salt MS with $0,1 / 4 \mathrm{MS}, 1 / 2 \mathrm{MS}, \mathrm{MS}$ with 5 replications. Hyperhydricity on patchouli shoots could be lowered, as indicated by the decrease in water content from $96 \%$ to $90-91 \%$, the increase in total chlorophyll content, and the increased number of palisade cells and stomata on the leaf treatment outcome. The concentration treatment of ammonium nitrate showed better results than the concentration of macros salt in increasing the total chlorophyll content, but it did not differ significantly in lowering water levels and increasing the number of palisade cells and stomata. $1 / 4 \times$ concentration treatment of ammonium nitrate could increase chlorophyll content of 0.16 to $0.97 \mathrm{mg} \cdot \mathrm{g}^{-1}$, but MS with $1 \mathrm{x}$ concentration showed the best result in the increase of number of palisade cells and stomata of the leaves.
\end{abstract}

Keywords: Ammonium nitrate, Hyperhydricity, Macro Salt, Shoot-tip culture.

\section{INTRODUCTION}

Patchouli (Pogostemon cablin Benth.) is one of the essential oil producing plants which gives the profit of foreign exchange for more than $50 \%$ of the total exports of Indonesian essential oil. The area of planting patchouli in Indonesia declined, in 2009 the total area of 24.536 ha turned to 23.635 ha in 2012. The decrease did not only occur in area of patchouli plant but also on the productivity of patchouli which in 2009, $113.27 \mathrm{~kg} . \mathrm{ha}^{-1}$ and in 2012, $87.20 \mathrm{~kg} \cdot \mathrm{ha}^{-1}$ [1]. Low productivity and oil quality were caused by the low quality of plant genetic due to the uncertain quality of seedlings and the development of various diseases [2].

Shoot-tip culture can be used to produce plants having virus-free, genetically homogenous and higher reproduction rate [3]. Plant propagation through shoot-tip culture is able to increase

\footnotetext{
${ }^{*}$ Correspondence author:

Aisyah Rahmawati Zen

Email : aisyah.chairul1116@gmail.com

Address : Master Program of Biology, University of Brawijaya, Veteran Malang, 65145
}

the rate of induction and multiplication of shoots, to improve the quality of produced seeds, however the content of nutrients and liquid medium can improve the hyperhydricity. Hyperhydricity on shoots culture results in leading to the decrease ability of plantlets regeneration [4].

Hyperhydricity is a condition of abnormal morphology and physiology which causes excessive hydration, low lignification, weakening of the stomata function, and lowering the mechanical strength of cultured plant tissue, leading to low regeneration of plantlets. Hyperhydricity symptoms are changes in plantlets conditions which becomes clear as a result of low levels of chlorophyll and high water content in plantlets. Shoots from culture experiencing hyperhydricity have thin cuticle layer, a reduced number of palisade cells, irregular stomata, impaired development of the cell wall, and large intercellular space on the mesophyll cells layer [4]. Several factors can cause hyperhydricity on shoots culture results, such as high salt concentration in the medium, 
high concentration of ammonium in the medium, low concentrations of gelling agent, the concentration of microelement and hormonal imbalance, relatively high humidity, low light intensities, the accumulation of gas in the culture bottle, as well as the type of explant used [5].

Salinity on MS medium leads to the top of Salix babylonica experience hyperhydricity and died while transferred to the medium with equal salinity. The decreased levels of $\mathrm{NH}_{4}^{+}$in the media are associated with the increased hyperhydricity and the decreased lignification on chestnut and willow plants [6]. Hyperhydricity on chestnuts that occures in MS medium can be prevented by using ammonium nitrate macronutrient in $1 / 2$ concentration [7]. The decreasing of ammonium nitrate concentration in the WP media of culture Amelanchier arborea increased the total number and percentage of shoots [8].

Shoot tip culture of patchouli usually has good multiplication of shoot, in the other hand most of shoot experience hyperhydricity which causes the decrease ability of plantlets regeneration. Since, hyperhydricity can be conducted by the high level of ammonium nitrate and macro salt in medium. Several studies have been done to reduce the hyperhydricity occurrence, by lowering the concentration of ammonium nitrate [7] and lowering the salt concentration in the media to $1 / 3$ concentration of MS [6]. Thus, modification on MS media need to be conducted with expectation patchouli planlet results in has a good regenerations ability.

\section{MATERIALS AND METHODS \\ Shoot tip culture}

Shoot tip used was taken from shoots of cultured patchouli leaves on solid MS medium (Murashige and Skoog) containing $0.1 \mathrm{mg} \cdot \mathrm{L}^{-1} \mathrm{NAA}$ and $0.3 \mathrm{mg} \cdot \mathrm{L}^{-1}$ BAP. Shoot tip on cultured patchouli shoots were planted in liquid MS medium and incubated in bright conditions at a temperature of $25^{\circ} \mathrm{C}$ for 6 weeks. Hyperhydricity shoots from shoot-tip culture results which had the same height and size were cultured on solid MS medium with a concentration of ammonium and macro salt of $0,1 / 4,1 / 2$, and $1 \times$ concentrations. The cultures were incubated in bright conditions at a temperature of $25^{\circ} \mathrm{C}$. 8 weeks-cultured patchouli shoots were evaluated concerning with its Hyperhydricity by measuring the level of water content, chlorophyll content, and the number of palisade cells and stomata of the leaves.

\section{Water and Chlorophyll Content of Planlet}

The water content was calculated based on the difference between wet weight and dry weight of shoots. Chlorophyll content was measured by 0.2 grams leaves which were homogenized and extracted with acetone then its absorbance was measured using a spectrophotometer at a wavelength of 647 and $665 \mathrm{~nm}$. Chlorophyll content was analyzed by Coombs and Hall method [9].

\section{Palisade and Stomata Count}

Making the section for counting palisade cells was done by making the crosswise slices of leaves using microtome. Making the section for the observation of the stomata number was done by clearing. Leaves were fixed using $70 \%$ ethanol for 24 hours, then clearing was performed by soaking the leaves in a solution of $5 \% \mathrm{NaOH}$ until leaves became clear and visible. Counting the number of palisade cells and stomata of the leaves was done under a microscope with a magnification of 400x.

This research was conducted in the experimental design used a randomized block design. Each treatment was repeated 5 times (bottle) and on each bottle were 4 shoots explants cultured. The obtained data were tested for normality data then performed statistical analysis using SPSS software for Windows 16 and if there was a difference, it would be followed by Tukey test at 5\% significance level.

\section{RESULTS}

\section{Water and Chlorophyll Content of Planlet}

Patchouli shoots experiencing hyperhydricity have a structure that looks clear (glassy). Clear green color in leaves that experienced hyperhydricity was caused by the deficiency of chlorophyll and the high water content. The water content in plants can be a marker of a hyperhydricity condition. High level of water is a hyperhydricity trait in plants. The water content of patchouli shoots experiencing hyperhydricity by $96 \%$, while the water content in normal shoots by $85 \%$. Cultured patchouli shoots in media through treatment with ammonium nitrate and macro salt had a tendency of decreased water content. The water content of shoots on media containing with ammonium and macro salts with various concentrations ranged between $90-91 \%$. This suggests that treatment with various concentrations of ammonium and macro salt media (Fig. 1a). 
Shoots experiencing hyperhydricity typically have low levels of chlorophyll. The chlorophyll content of patchouli leaf shoots in hyperhydricity was $0.16 \mathrm{mg} \cdot \mathrm{g}^{-1}$ while in normal patchouli leaf was $1.5 \mathrm{mg} \cdot \mathrm{g}^{-1}$. Total of chlorophyll content of patchouli leaves shoots from cultures on MS medium with ammonium nitrate concentration treatment showed an increase compared to those on leaf experiencing hyperhydricity, meanwhile the treatment $1 / 4$ and $1 / 2$ of macro salt concentration showed the results of total chlorophyll content tends to not differ between treatments. The concentration treatment of ammonium nitrate on MS medium produced patchouli leaf shoots with higher chlorophyll content than all of macro salt concentration in the media. The highest chlorophyll content of patchouli shoots leaves was demonstrated by culture results on the concentration treatment of ammonium nitrate into $1 / 4 \times$ which was $0.97 \mathrm{mg} \cdot \mathrm{g}^{-1}$ (Fig.1b).

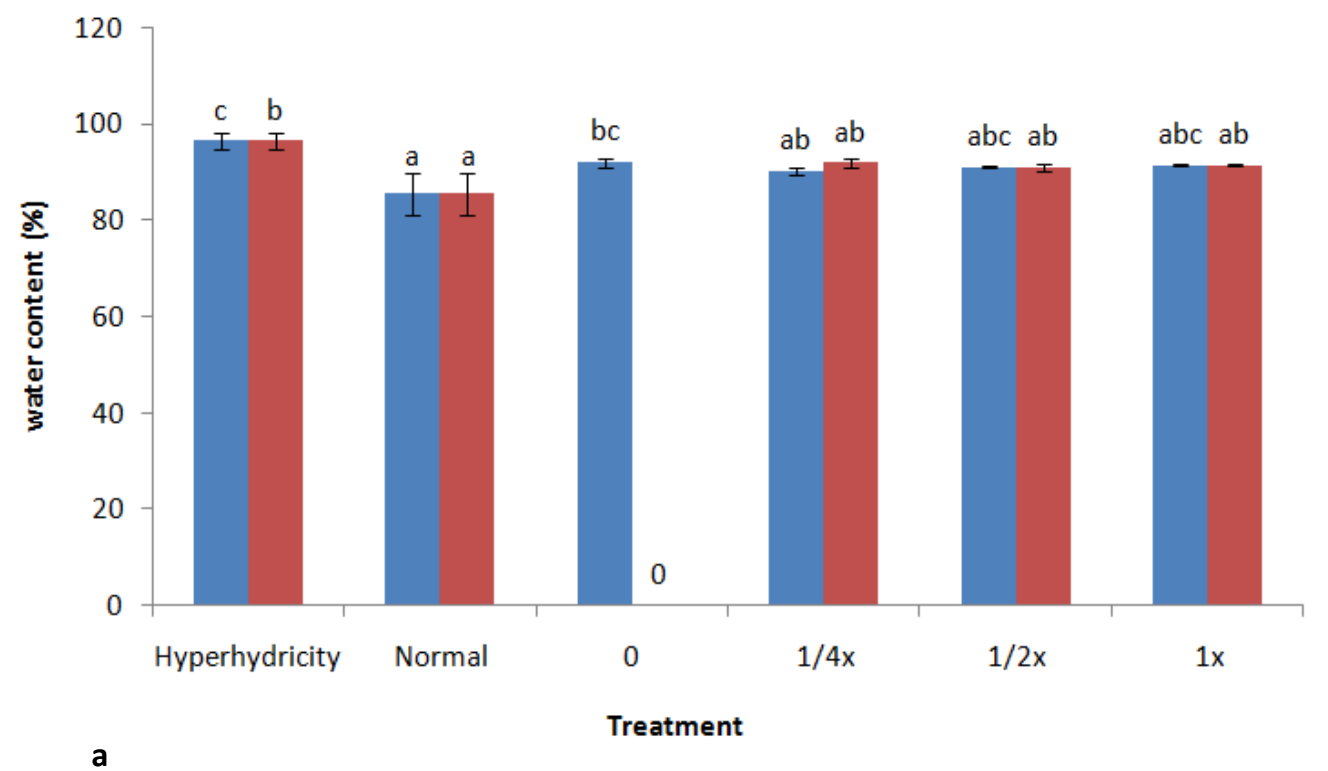

n Ammonium Concentration $\quad$ Macro Salt Concentration

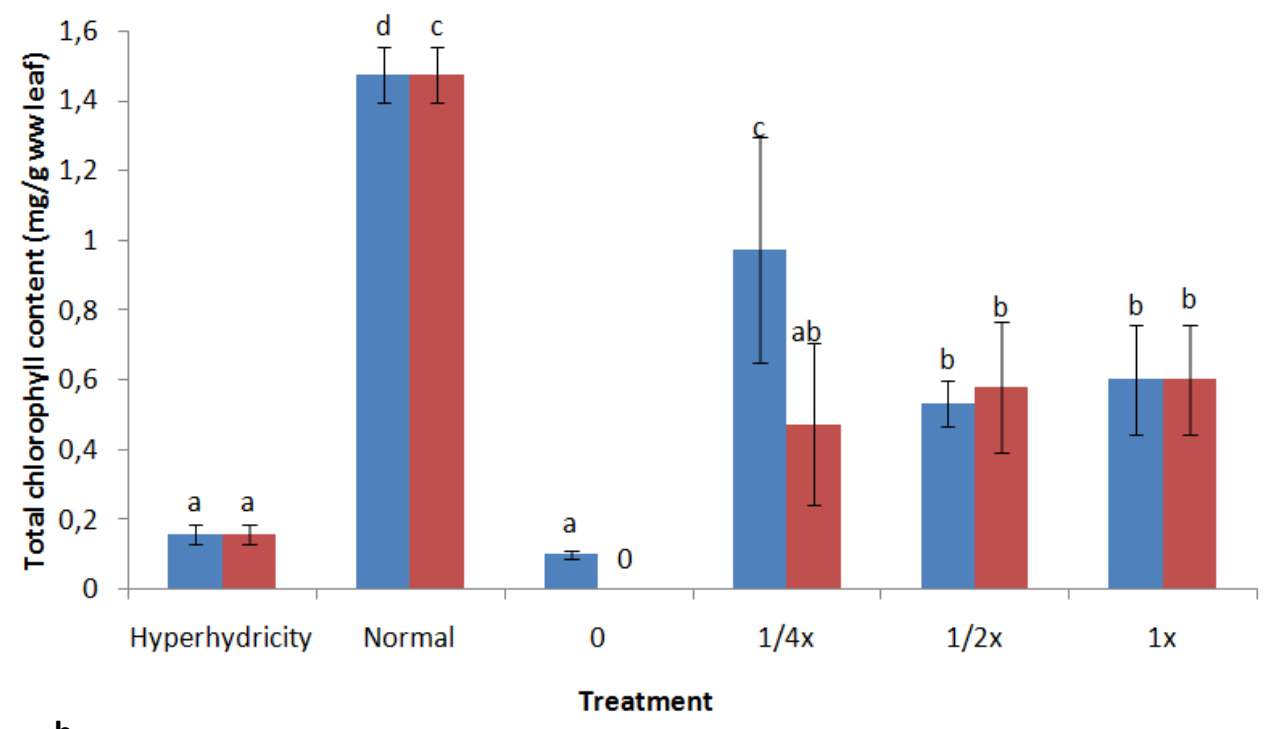

b

- Ammonium concentration Macro Salt Concentration

Figure 1. Comparison of water content and chlorophyll content in patchouli shoots experiencing hyperhydricity, normal, and after treatment with the concentration of ammonium nitrate and salt macro on MS medium.

a. water content, $\mathbf{b}$. total chlorophyll content.

The same letter in the same treatment showed no difference in treatment results in further test of Tukey. 


\section{Palisade and Stomata of Planlet's Leaf}

Patchouli leaves experiencing hyperhydricity had palisade cells which was larger than the palisade cells in normal patchouli leaves. Patchouli leaves experiencing hyperhydricity had a wide space among cells. The composition of palisade cells in the Patchouli leaves experiencing hyperhydricity was more tenuous and not uniform in size. They had less palisade cell than normal leaves (Fig. 2a). Normal plantlets patchouli leaves seemed to have palisade cells which were well-ordered, dense, homogenous, and had narrow space among cells (Fig. 2b). The number of stomata on the leaves experiencing hyperhydricity was less than in normal leaves and after treatment. Stomata on the patchouli leaves experiencing hyperhydricity appeared to have abnormal stomata and guard cells changing shape. Stomata experienced malformations with guard cells which became more elongated than the guard cells in normal patchouli leaves (Fig. 2e). Normal patchouli leaves had rounded stomata and guard cells (Fig. 2f).

Patchouli shoots leaf on the concentration treatment of $1 / 4$ ammonium and $1 / 2$ macro salt concentrations showed characteristics similar to normal patchouli leaves. Both had a palisade cell structure which began to be well-ordered, dense, and homogenous. Both treatments still indicated a space among cells that was wider than normal patchouli leaves (Fig. 2c and 2d). Patchouli leaves coming from the results of the treatment with MS medium containing with $1 / 4$ ammonium concentrations and $1 / 2$ macro salt concentrations appeared to have shape of stomata and guard cells which were similar to normal stomata shape (Fig. $2 \mathrm{~g}$ and $2 \mathrm{~h}$ ).

Treatment variations in the concentration of ammonium nitrate showed the better results in increasing the number of palisade cells of leaves than treatment variations in the concentration of salt macro, but the treatment $1 x$ concentrations of ammonium nitrate and macro salt demonstrated superior results in increasing the number of cells palisade from 12 to 26 (Fig. 3a) compared to treatment with concentrations of $0,1 / 4$ and $1 / 2 x$. The number of stomata of the cultured patchouli leaves shoot on MS medium with the treatment variations of ammonium nitrate and macro salt indicated that there were an increasing number of stomata compared to the leaves undergoing hyperhydricity, but the treatment $1 x$ concentrations of ammonium nitrate and macro salt showed the highest results of the number of stomata by 64 (Fig. 3b).
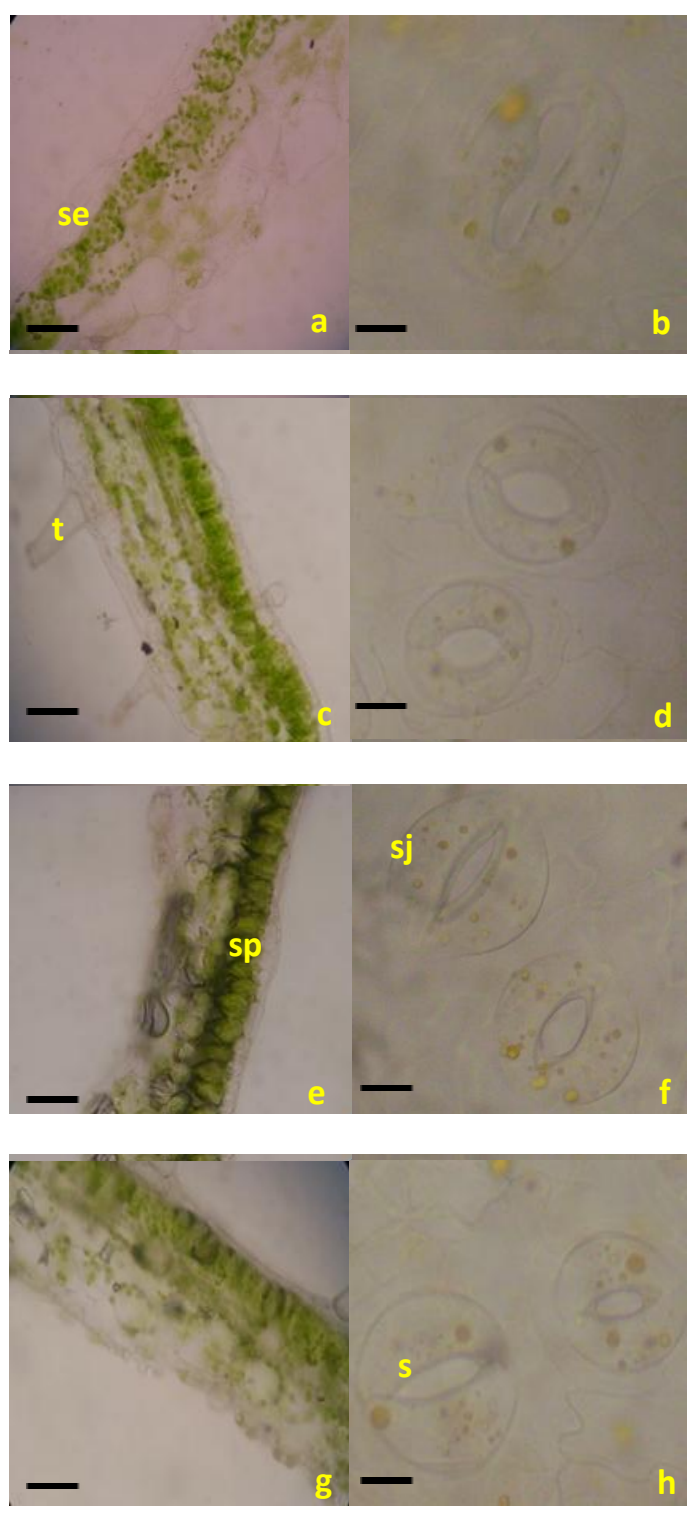

Figure 2. Structure of the leaves anatomy with and without hyperhydricity on patchouli shoots after treatment.

a,b. the cross section and stomata of the leaves in hyperhydricity;

c,d. the cross section and the stomata of the normal leaves;

e,f. cross-section and the stomata of the leaves as the results of the treatment on MS medium with $1 / 4$ ammonium concentration;

g,h. cross-section and stoma-ta of leaves as the results of treatment on MS medium with macro salt concentration.

se: epidermis; t: trichomes; sp: palisade cells; sj: guard cells; s: stomata. Bar: $15 \mu \mathrm{m}$. 


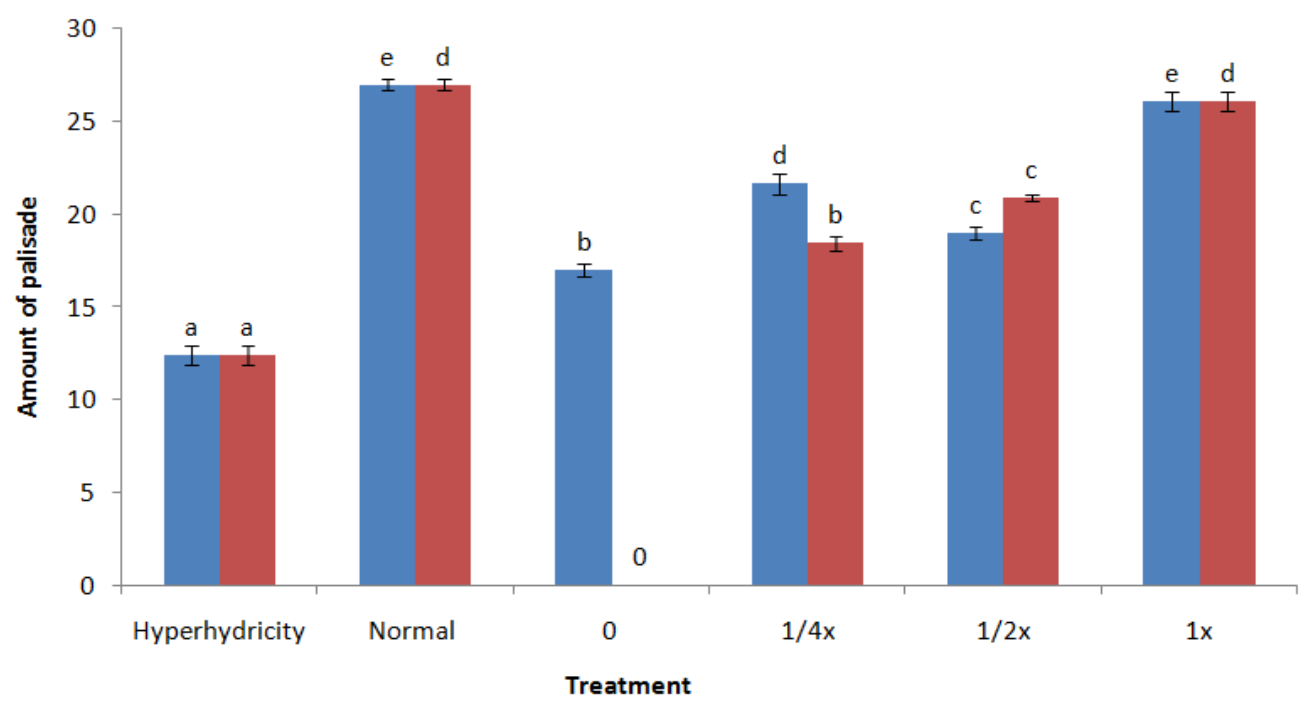

a

$$
\text { Ammonium concentration } \quad \text { Macro salt concentration }
$$

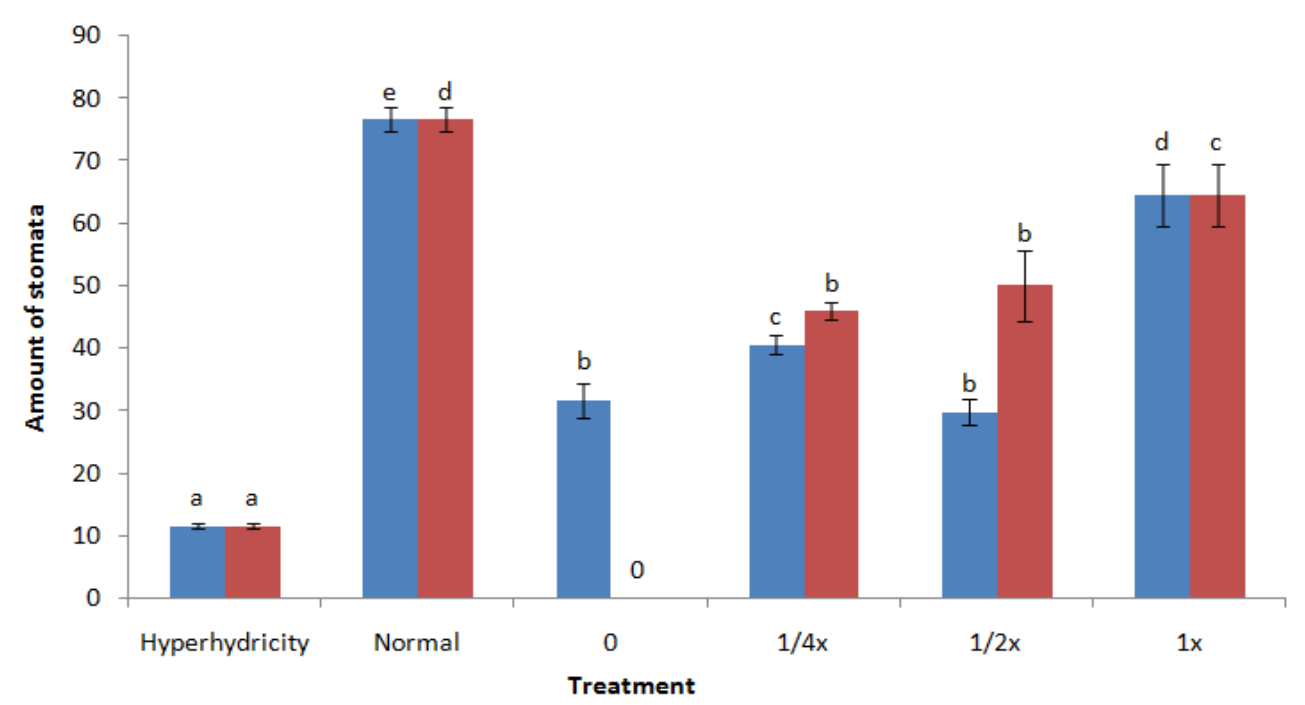

b

Ammonium concentration Macro salt concentration

Figure 3. Comparison of the number of palisade cells and stomata on patchouli shoots leaves in the Hyperhydricity, normal, and after treatment with the concentration of ammonium and macro salt on MS medium.

a. the number of palisade cells, $\mathbf{b}$. the number of stomata.

The same letter in the same treatment showed no difference in treatment results in further test of Tukey.

\section{DISCUSSION}

\section{Water and Chlorophyll Content of Planlet}

High water levels in liquid media caused the plant suffering hyperhydricity. The results showed that the plant suffered hyperhydricity had a high water content and it was allegedly also associated with low levels of chlorophyll in the leaves. Accumulation of water content caused the formation of intercellular space that was wider at the mesophyll layer so that the number of palisade cell became less than normal leaves. The small number of palisade cells is assumed to cause the low chlorophyll content, because most of the chloroplasts in the leaves lied in the palisade cells in the mesophyll layer [10].

The chlorophyll content in the patchouli shoots undergoing hyperhydricity was lower than 
in the normal leaves shoots and after treatment. The low levels of chlorophyll was allegedly due to a lack of chloroplasts in leaf shoots experiencing hyperhydricity. The chlorophyll content in the leaves undergoing hyperhydricity was significantly lower than in normal leaves. A previous study reported that hyperhydricity caused a decrease in the number of chloroplasts and the ultrastructural analysis showed the damage to the thylakoid membranes caused by hyperhydricity [5]. Low levels of chlorophyll in the leaves undergoing hyperhydricity were allegedly caused by the lack of appropriate concentrations of nutrients in the media. Levels of chlorophyll in plants were influenced by several factors, such as light, sugar or carbohydrates, water, temperature, genetic factors and nitrogen elements, magnesium, iron, manganese, $\mathrm{Cu}, \mathrm{Zn}$, sulfur, and oxygen. It could be used as the basis that the right macro salts comparison can keep levels of chlorophyll in a plant remained normal [11]. The findings of this study indicated that the subcultured patchouli shoots on MS medium with $1 / 4 x$ ammonium had higher levels of chlorophyll than the other treatments. The findings of this study were similar to previous research which reported that the reduction in ammonium concentrations in WPM media of apricot culture produced shoots that had a high chlorophyll content and free hyperhydricity [8].

\section{Palisade and Stomata of Planlet's leaf}

The results of this study indicated that the number of palisade cells in the leaves experiencing hyperhydricity was less than in the normal leaves. The small number of palisade cells in the leaves experiencing hyperhydricity was related to changes in the structure of palisade cell which was larger than in normal leaves and the high water content in the leaves experiencing hyperhydricity. The larger size of a cell, the lower number per unit area. The high water content in leaves experiencing hyperhydricity resulted in the accumulation of water on the leaves, causing the wider space among cells.

Changes in the structure of the palisade cells in leaves experiencing hyperhydricity on this study were believed to be related to the mechanism of cell wall formation [12]. The formation of a cell wall in hyperhydricity cell was impaired. Cells undergoing hyperhydricity looked like protoplasts having very thin cell walls. The size became larger and abnormal form of palisade cells in the leaves undergoing hyperhydricity confirmed a relation between hyperhydricity and the disruption of cell wall formation. The disruption of cell wall formation was allegedly due to lack of lignin synthesis [13]. Lignin is synthesized in the cell walls, so the lack of lignin synthesis results in cell walls undergoing hypolignification. Lignin synthesis is affected by a number of enzymatic activity. A decrease of the enzyme performance in the metabolism of phenols, including the reduction of phenylalanine ammonia lyase and increased activity of glutamate dehydrogenase resulted in hypolignification. The increased glutamate dehydrogenase activity was influenced by the concentration of ammonium nitrate in the media [13].

Hyperhydricity was aassumed to be associated with abnormal stomata structure and the declining number of stomata. Abnormal stomata structure which was visible from the modified shape of neighboring cells and the small number of stomata could be assumed to cause regulation of water on the leaves became inefficient, especially in the transpiration process which resulted in the accumulation of water [14]. Accumulation of water on the lacunae leaves caused the hyperhydricity. Accumulation of water in plants that undergo this hyperhydricity was assumed to affect the water content in plants. This was proved by higher levels of water in the plantlets which were experiencing hyperhydricity than in the normal plantlets and after treatment [14].

The amount and structure of palisade cell from treatment results with ammonium and macro salt in MS media seemed to have improved in the structure and the number of palisade cells was similar to the normal leaf, but the treatment with $1 x$ concentration of ammonium and macro salt showed the best results compared to the other treatments. Similar results were also shown in the number of stomata parameters. These results indicated that treatment variations and concentrations of ammonium and macro salt did not have significant effect on the increase of the number of palisade cells and stomata of the leaves. The increasing number of palisade cells and stomata of the leaves was allegedly associated with removing shoots from liquid medium to solid medium. Solid media was assumed to affect the decrease of hyperhydricity in several studies. The seaweed on the media could bind the water to the media so that water absorbed by the explant was not too much. The seaweed was also assumed to modify the availability of dissolved 
nutrients in the media through a chemical reaction so that the nutrients absorbed by explant not excessive [15].

\section{CONCLUSION}

Subculture of patchouli shoots undergoing hyperhydricity on MS medium could only lower the hyperhydricity of shoots which was indicated by the decrease of water levels, increase of chlorophyll levels, and the number of palisade cells and stomata. The decrease of hyperhydricity was affected by the concentration of ammonium nitrate and macro salt on MS medium. The best decrease hyperhydricity of patchouli shoots was on MS medium with $1 x$ concentrations of ammonium nitrate and macro salt. The highest increase of total chlorophyll content in the media was at the concentration of $1 / 4 \mathrm{x}$ ammonium.

\section{REFERENCES}

[1] Setiawan, R. Rosman. 2013. Reducing productivity of patchouli. Warta Penelitian dan Pengembangan Tanaman Industri. 19(3). 8-11.

[2] Nasrun, Y. Nuryani, Hobir, Repianyo. 2004. Resistant selection of patchouli to the disease caused by Ralstonia solanacearum in planta. Journal Stigma. XII(4). 421-473.

[3] Gunaeni, E. 2008. Meristem culturer and antiviral ribavirin on Potato Plant. Agrivigor. 7(2). 105-112.

[4] Ivanova, M.V. 2009. Regulation of hyperhydricity in Aloe polyphylla propagated in vitro. PhD Thesis. Research Centre for Plant Growth and Development School of Biological and Conservation Sciences, University of KwaZulu-Natal. Pietermaritzburg.

[5] Chakrabarty, D., S.Y. Park, M.B. Ali, K.S. Shin, K.Y. Paek. 2005. Hyperhydricity in apple: ultrastuctural and physiological aspects. Tree Physiol. 26. 377-388.

[6] Letouzé, R., F. Daguin. 1987. Control of vitrification and hypolignification process in Salix babylonica cultured in vitro. Acta Horticulturae. 212: 185-191.

[7] Chauvin, J., G. Salesses. 1988. Advances in chestnut micropropagation (Castanea sp.). Acta Horticulturae. 227. 340-345.

[8] Brand, M. H. 1993. Agar and ammonium nitrate influence hyperhydricity, tissue nitrate and total nitrogen content of serviceberry (Amelanchier arborea) shoots in vitro. Plant Cell. Tiss. Organ Cult. 35. 203209.
[9] Coombs, J., D.O. Hall. 1987. Techniques in Bioproductivity and Photosynthesis. Pergamon Press. Oxford, U.K.

[10] Yu, Y., Y.Q. Zhao, B. Zhao, S. Ren, Y.D. Guo. 2010. Influencing factors and structural characterization of hyperhydricity of in vitro regeneration in Brassica oleracea var. Italica. Canadian J. Plant Sci. 91. 159-165.

[11] Hendriyani, I.S., N. Setiari. 2009. Chlorophyll content and long bean growth (Vigna sinensis) at different level of water avaliability. J. Sains Mat. 17(3). 145-150.

[12] Shreedar, R.V., L. Venkatachalam, B. Neelwarne. 2009. Hyperhydricity-related morphologic and biochemical changes in Vanilla (Vanilla planifolia). J. Plant Growth Regul. 28. 46-57.

[13] Rasco, S.M. 1997. In vitro vitrification (Hyperhydricity) In Shallot (Alium cepa var. gr. Aggregatum). Phillipp J. Crop Sci. 22(1). 14-22.

[14] Yasseen, Y.M., T.L. Davenport, W.E. Splittstoesser. 1992. Abnormal stomata in vitrified plants formed in vitro. Florida Agr. Exp. Station J. 105. 210-212.

[15] Abdoli, M. 2007. Effects of cultivar and agar concentration on in Vitro shoot organogenesis and hyperhydricity in Sunflower (Helianthus annuus L.). Pak. J. Botany. 39(1). 31-35. 